\title{
Magnetic resonance imaging and Psychiatry: past, present and future
}

\author{
Imagem por ressonância magnética e Psiquiatria: passado, presente e futuro
}

Michael John Brammer, BSc PhD

Professor of Neuroimaging, King's College London - London, United Kingdon; Head of Brain

Image Analysis Unit, Centre for Neuroimaging Sciences - King's College London - London,

United Kingdon.

It is now 35 years since the first magnetic resonance imaging (MRI) scan of a human body was performed ${ }^{(1)}$ and 20 years since functional MRI (fMRI) appeared as an experimental imaging tool ${ }^{(2)}$. When my colleagues and I began to use these two techniques in 1994, at the Institute of Psychiatry in London, it seemed that MRI was on the verge, radically changing our view of mental illness by allowing us to define what we often termed the "neural correlates" as psychiatric disorders. Only 10 years later, some of my colleagues ${ }^{(3)}$, in a book entitled Neuroimaging in Psychiatry were able to report hundreds of papers published using MRI to investigate a wide range of psychiatric disorders. However, only 6 years later, Bullmore et al. ${ }^{(4)}$ warned that Psychiatry could not afford to be "neurophobic" and that neuroscientific approaches, such as those using MRI, had a valuable part to play in advancing in this area of Medicine. By 2009, thousands of papers had appeared in high-impact psychiatric journals, such as the British and American Journals of Psychiatry, Archives of General Psychiatry, Molecular Psychiatry and Biological Psychiatry, as well as in Nature and Science. Clearly though, many in the psychiatric community remained to be convinced that MRI was proving (or would prove) to be a useful tool of clinical relevance.

It is possible to identify a number of reasons why this view might be held. Firstly, especially in the case of fMRI, there are issues about reproducibility. These arise from a number of sources. The main method of imaging "brain activation" used in fMRI is dependent on changes in the images related to blood oxygenation level (so called BOLD or Blood Oxygenation Level Dependent contrast). This technique produces relatively small changes in signal and is very prone to artifacts arising from many sources, some machine dependent, some due to respiration or heartbeat and some due to subject motion. Dealing with these artifacts requires complex image processing steps which most users probably do not fully understand and which can be implemented in a large number of different ways in standard processing packages such as AFNI, SPM, FSL or Brain Voyager (multiple processing possibilities are even available within each package). A combination of these factors, coupled with the tendency for studies to be carried out on small numbers of subjects (frequently less than 20) has led to the impression that results vary widely from research group 
to research group and from study to study. A second issue is related to this. Most studies to date using the packages listed above have concentrated on grouplevel analysis. This arises from the fact that the analysis is most usually carried out on each voxel (the smallest part of the brain that can be imaged) separately. As the effects are typically small (see above), this means that there is normally not enough power to make reliable statements about individual subjects. In terms of clinical application, group studies can clearly be useful. They can give information about "typical" changes in brain structure or function in a population of schizophrenic patients compared with controls, for example. This in turn can be used to identify which brain networks are involved and consequently, how drugs or cognitive interventions can be targeted and developed. However, in Europe and North America, such population level intervention strategies are not in step with Health Economics imperatives. The strategy of targeting a whole group in the same way when we know that they will not all respond favorably is being superseded by the philosophy of "Personalized Medicine". This aims to make the best use of money (important in Europe and the United States in a time of economic crisis) by targeting individuals, making the most effective use of resources and giving better patient outcomes.

In the last decade, a rethink of the way in which MRI data are utilized has begun to make it possible to use imaging data in the framework of personalized medicine. The main change required is to use the imaging data in such a way as to facilitate individual subject prediction. The methods used to achieve this are well-known in computer science and mathematics and are often called "machine learning" methods. In the most common methodologies used in MRI, this means that a "machine" (in this case a computer program) "learns" how to associate brain imaging data with a "data class". As an example, the "machine" might learn to find the best way to distinguish between diagnostic categories (e.g. patient/control). Once this has been learned using a training data set it can be applied to individual test data sets to assign them to a category. In other words, the computer program can make an individual diagnosis. For some examples of this in use see Kloppel et al., Ecker et al. ${ }^{(5,6)}$. It seems that this development in the manner how imaging data are used may go some way towards achieving the aims of two decades ago, namely making imaging useful in Psychiatry. In fact, the situation may be becoming even more interesting. The simple use of machine learning as an extra method to assist diagnosis of psychiatric disorders may be useful but we may not have come close to realizing the full potential of the technique. Recent studies using "baseline" scans followed by psychiatric follow-up have shown that it is possible to predict, in psychosis and depression ${ }^{(7)}$, some aspects of the future trajectory of the disorder before clinical manifestations are detectable (using baseline imaging data). This moves us further towards the goal of maximizing the effectiveness (in clinical and cost terms) of intervention and making better use of imaging data.

\section{REFERENCES}

1. Damadian R, Goldsmith M, Minkoff L. NMR in cancer: XVI. FONAR image of the live human body. Physiol Chem Phys. 1977;9(1):97-100, 108.

2. Ogawa S, Tank DW, Menon R, Ellermann JM, Kim SG, Merkle H, et al. Intrinsic signal changes accompanying sensory stimulation: functional brain mapping with magnetic resonance imaging. Proc Natl Acad Sci U S A. 1992; 89(13):5951-5

3. Fu $\mathrm{CH}$, Senior $\mathrm{C}$, Russell TA, Weinberger D, Murray R. Neuroimaging in psychiatry. London: Martin Dunitz; 2003.

4. Bullmore E, Fletcher P, Jones PB. Why psychiatry can't afford to be neurophobic. Br J Psychiatry. 2009;194(4):293-5

5. Klöppel S, Stonnington CM, Barnes J, Chen F, Chu C, Good CD, et al. Accuracy of dementia diagnosis: a direct comparison between radiologists and a computerized method. Brain. 2008;131(Pt 11):2969-74.

6. Ecker C, Rocha-Rego V, Johnston P, Mourao-Miranda J, Marquand A, Daly EM, Brammer MJ, Murphy C, Murphy DG; MRC AIMS Consortium. Investigating the predictive value of whole-brain structural MR scans in autism: a pattern classification approach. Neuroimage. 2010;49(1):44-56.

7. Mourão-Miranda J, Oliveira L, Ladouceur CD, Marquand A, Brammer M, Birmaher $B$, et al. Pattern recognition and functional neuroimaging help to discriminate healthy adolescents at risk for mood disorders from low risk adolescents. PLoS One. 2012;7(2):e29482. 